\title{
Chemotactic Factors Regulate Lectin Adhesion Molecule 1 (LECAM-1)- dependent Neutrophil Adhesion to Cytokine-stimulated Endothelial Cells In Vitro
}

\author{
C. Wayne Smith," Takashi Kei Kishimoto," Omid Abbass," Bonnie Hughes," Robert Rothlein," \\ Larry V. Mclntire," Eugene Butcher, ${ }^{\star}$ and Donald C. Anderson ${ }^{\star}$ \\ ${ }^{*}$ Section of Leukocyte Biology, Department of Pediatrics, Baylor College of Medicine, Houston, Texas 77030; ${ }^{\ddagger}$ Department of Pathology, \\ Stanford University School of Medicine, Stanford, California 94305, ${ }^{8}$ Biomedical Engineering Laboratory, Rice University, \\ Houston, Texas 77251; and "Boehringer Ingelheim Pharmaceuticals, Inc., Ridgefield, Connecticut 06877
}

\begin{abstract}
Monoclonal antibodies recognizing CD18, CD11a, CD11b, and neutrophil lectin adhesion molecule 1 (LECAM-1), i.e., the human homologue of the murine MEL-14 antigen, were used to assess the relative contribution of these glycoproteins to neutrophil-endothelial adhesion. Under static conditions, the adhesion of neutrophils to IL-1-stimulated human umbilical vein endothelial cell (HUVEC) monolayers was inhibited by antibodies to CD18, CD11a, and the neutrophil LECAM-1, and the effect of combining anti-LECAM-1 and anti-CD11a was almost additive. Under flow at a wall shear stress of $1.85 \mathrm{dyn} /$ $\mathrm{cm}^{2}$, a condition where CD18-dependent adhesion is minimal, anti-LECAM-1 inhibited adhesion by $>50 \%$. Chemotactic stimulation of neutrophils induced a rapid loss of LECAM-1 from the neutrophil surface, and the level of neutrophil surface LECAM-1 was closely correlated with adhesion under flow. Neutrophils contacting the activated endothelial cells for $\mathbf{3 0}$ min lost much of their surface LECAM-1, a phenomenon induced by a soluble factor or factors released into the medium by the stimulated monolayers, and a high percentage migrated through the HUVEC monolayer. This migration was almost completely inhibited by anti-CD18, but was unaffected by antibodies to neutrophil LECAM-1. These results support the concept that LECAM-1 is a neutrophil adhesion molecule that participates in the adherence of unstimulated neutrophils to cytokine-stimulated endothelial cells under conditions of flow, and is then lost from the neutrophil surface coincident with the engagement of CD18-dependent mechanisms leading to transendothelial migration. (J. Clin. Invest. 1991. 87:609-618.) Key words: adhesion $\bullet$ CD18 $\bullet$ chemotactic factors $\bullet$ interleukin $1 \cdot$ monoclonal antibodies
\end{abstract}

\section{Introduction}

The adherence of human neutrophils to human endothelial cells under static conditions in vitro is increased by chemotactic stimulation of the neutrophils $(1-10)$, or by stimulation of the endothelial cells with endotoxin or cytokines, e.g., IL-1 $\beta(4$, $6-9,11-15)$. Studies using monoclonal antibodies indicate that

Address reprint requests to Dr. Smith, Texas Children's Hospital Clinical Care Center, 8080 N. Stadium Drive, Suite 2100, Houston, TX 77054 .

Received for publication 18 July 1990 and in revised form 18 Sep tember 1990

J. Clin. Invest.

(c) The American Society for Clinical Investigation, Inc.

0021-9738/91/02/0609/10 \$2.00

Volume 87, February 1991, 609-618 the adherence of stimulated neutrophils is dependent on both CD1 1a/CD18 (LFA-1) and CD11b/CD18 (Mac-1) (4, 5, 7, 9, $10)$, but the adherence of unstimulated neutrophils to stimulated endothelial cells appears to be more complex. It depends on endothelial intercellular adhesion molecule 1 (ICAM-1) ${ }^{1}$ interacting with CD1 la/CD18 (LFA-1) on the neutrophil (6), and endothelial-leukocyte adhesion molecule 1 (ELAM-1) (16, 17) interacting with an unknown, $C D 18$-independent structure on the neutrophil (18). Recently, evidence was obtained that the human homologue of the murine MEL-14 antigen $(19,20)$ on the neutrophil, identified by MAbs DREG-56 (21), Leu8, and TQ1 $(22,23)$ or anti-LAM-1 $(23)$, is also involved and interacts with an unknown structure on the endothelial cell (23a). The roles these various molecules play in the neutrophil functions enhanced by or requiring adhesion are poorly defined. While evidence exists that Mac- 1 is involved in adherence-dependent $\mathrm{H}_{2} \mathrm{O}_{2}$ production $(24,25)$, and LFA-1, Mac-1, and ICAM-1 are necessary for optimum transendothelial migration $(7,9)$, the functional contributions (other than adhesion) of ELAM-1 and the MEL-14 antigen, both members of the lectin-cellular adhesion molecule (LEC-CAM) family of adhesion molecules (26), are unknown.

Monoclonal antibody MEL-14 and MAbs to LFA-1 and Mac-1 markedly reduce mouse neutrophil extravasation to inflamed tissues in vivo $(27,28)$. We have observed that chemotactic stimulation causes a rapid shedding of the LEC-CAM from the surface of murine neutrophils (29) and human neutrophils (30). This rapid down-regulation occurs coordinately with rapid upregulation of Mac-1 expression and function, which effectively turns off one adhesion mechanism while turning on another mechanism. The loss of neutrophil LECCAM and the increase in Mac-1 is observed not only in vitro, but also in vivo in neutrophils in the process of extravasating in inflammatory sites $(28,29)$. These results suggest that Mac-1 and LEC-CAM on the neutrophil surface mediate fundamentally distinct adhesion events. We have proposed that neutrophil lectin adhesion molecule 1 (LECAM-1) is critical for the initial binding event between unstimulated neutrophils in circulation and the inflamed endothelium, whereas LFA-1 and Mac-1 may be more important for adhesion strengthening and transendothelial migration. In this article we directly test this hypothesis in an in vitro model examining adhesion under shear stress, transendothelial migration, and neutrophil activation. Since a MAb (DREG-56) to human LECAM-1 that

1. Abbreviations used in this paper: ELAM-1, endothelial-leukocyte adhesion 1; HUVEC, human umbilical vein endothelial cells; GMCSF, granulocyte/monocyte colony-stimulating factor; ICAM-1, intercellular adhesion molecule 1; LECAM-1, lectin adhesion molecule 1 ; LEC-CAM, lectin-cellular adhesion molecules; PAF, platelet-activating factor; PE, phycoerythrin. 
blocks the lectin activity of this molecule on human neutrophils and lymphocytes (30) has recently been developed (21), these studies were performed using human cells.

\section{Methods}

Isolation of polymorphonuclear leukocytes. Neutrophils were obtained from healthy adult individuals and three patients with the severe phenotype of CD18 deficiency (31) were purified from citrate anticoagulated, dextran-sedimented venous blood samples over Ficoll-Hypaque gradients and were suspended in Dulbecco's phosphate-buffered saline (PBS; Gibco Laboratories, Grand Island, NY), pH 7.4, containing $0.2 \%$ dextrose as described (7). Neutrophils were maintained at $4^{\circ} \mathrm{C}$ in PBS for up to $4 \mathrm{~h}$ at a concentration of $10^{7} / \mathrm{ml}$.

Monoclonal antibodies. MAbs used in these studies included preparations of $\mathrm{IgG}$, and $\mathrm{F}\left(\mathrm{ab}^{\prime}\right)_{2}$ fragments. The anti-CD18 MAb, R15.7 (IgG1) (32) and anti-CD1 la MAb, R3.1 (IgG1) (7) were prepared as previously described. Anti-CD18 MAb TS1/18 (33) was generously provided by T. Springer, Center for Blood Research, Boston, MA. The anti-CD1 lb MAbs, 904 (IgG1) (34) and Leu 15 (anti-CD1 lb, tagged with phycoerythrin [PE]) were obtained from Coulter Electronics, Inc., Hialeah, FL, and Becton, Dickinson \& Co., Mountain View, CA, respectively. One anti-neutrophil MAb 4A5 (IgGl) was prepared as previously described (7), and used as a control in the adherence and migration assays. It has been evaluated in our laboratory for the ability to inhibit neutrophil adherence to endothelial cells, and found to be without effect. A MAb to the human homologue of the MEL-14 antigen, DREG-56 (IgG1) was prepared as previously described (21), and another MAb with this specificity, Leu8 tagged with FITC, was purchased from Becton, Dickinson \& Co. All MAbs that bind to neutrophils were titered using flow cytometry (FACScan, Becton, Dickinson $\&$ Co.) to determine the concentration that saturated surface binding sites of unstimulated and stimulated cells (i.e., stimulation with $10 \mathrm{nM}$ FMLP, $37^{\circ} \mathrm{C}, 15 \mathrm{~min}$ ). In most functional studies, MAbs were initially used at twice the saturating concentration.

Immunofluorescence flow cytometry. The quantitation of $\mathrm{MAb}$ binding to neutrophils involved the use of fluorescein isothiocyanate (FITC)- or PE-tagged antibodies. In some instances, the fluorochrome was directly attached to the primary antibody, in others it was attached to a $\mathrm{F}\left(\mathrm{ab}^{\prime}\right)_{2}$ goat anti-mouse second antibody for indirect detection of the primary antibody binding. Analysis was performed on a FACScan, and results were expressed as mean relative linear fluorescence.

Studies designed to assess the kinetics of change in the surface antigen recognized by a specific antibody after chemotactic stimulation of the neutrophils were performed in two ways. In one protocol, neutrophils were suspended in $0.1 \mathrm{ml} \mathrm{PBS}$ and exposed at $37^{\circ} \mathrm{C}$ to the stimulus (e.g., $10 \mathrm{nM}$ FMLP). At the specified time, the cell suspension was diluted $100 \times$ in ice-cold PBS and maintained at ice bath temperatures during the subsequent preparation of the cells for flow cytometry, i.e., tagging with the appropriate primary and secondary antibodies, and fixation in 5\% paraformaldehyde as previously described (7). In some cases, a second protocol was used to assess the changes occurring within the first few minutes after stimulation: neutrophils were suspended for 10 min at $37^{\circ} \mathrm{C}$ in PBS containing the primary antibody (directly labeled with the fluorochrome) at saturating concentrations. This preparation was then directly sampled in the flow cytometer to determine the baseline level of fluorescence of these viable cells. The chemotactic stimulus was then added and the suspension was sampled to determine the changes in fluorescence by drawing 3,000 viable cells into the flow cytometer at one minute intervals. Temperature was maintained at $37^{\circ} \mathrm{C}$ using a air curtain incubator, and cells were suspended by gentle vortexing.

The following procedure was used to estimate the number of binding sites for antibody FITC-Leu-8 on the neutrophil surface. Simply Cellular microbeads (Flow Cytometry Standards Corp., Research Triangle Park, NC) were stained identically to and in parallel with neutrophils. These $8-\mu \mathrm{m}$ diam beads have covalently bound goat anti-mouse antibodies on their surface with a known number of binding sites per bead. Analysis was performed using a FACScan flow cytometer calibrated with quantitative FITC microbead standards (Flow Cytometry Standards Corp.) to determine the fluorescence/protein ratio. Using this ratio and the quantitative FITC standards, the number of binding sites for these antibodies on neutrophils could be estimated.

The chemotactic stimuli were FMLP (Sigma Chemical Co., St. Louis, MO), recombinant endothelial IL-8 (Bachem, Inc., Torrance, $\mathrm{CA}$ ), and platelet-activating factor (PAF; Sigma Chemical Co.).

Preparation of human umbilical vein endothelial cells (HUVEC). The cells were harvested and characterized as to acetylated low-density lipoprotein (acLDL) binding and factor VIII expression according to established techniques as previously described (7). Cells from 5-10 umbilical cords were pooled and plated in RPMI 1640 containing $10 \%$ fetal calf serum (FCS) and $10 \%$ bovine serum, antibiotics, heparin $(0.1$ $\mathrm{mg} / \mathrm{ml})$, and endothelial cell growth factor $(0.05 \mathrm{mg} / \mathrm{ml})$, and maintained for $3-4 \mathrm{~d}$ at $37^{\circ} \mathrm{C}, 5 \% \mathrm{CO}_{2}$ humidified atmosphere. Visually confluent monolayers on gelatin $(0.1 \%)$ coated $25-\mathrm{mm}$ round or 24 50-mm rectangular glass coverslips were prepared from first passage cells harvested with $0.05 \%$ trypsin and $0.02 \%$ EDTA in PBS. HUVEC were pretreated with various concentrations of interleukin 1 (IL-1; Genzyme, Boston, MA), in most instances for 3-4 h, a time previously shown to be optimal for the enhancement of neutrophil adherence (6).

HUVEC-conditioned supernatants were prepared from HUVEC monolayers that were either unstimulated or stimulated with IL-1 for up to $5 \mathrm{~h}$. The monolayers were rinsed by dipping five times in two changes of PBS and then overlaid with $0.3 \mathrm{ml}$ of PBS for $1 \mathrm{~h}$ at $37^{\circ} \mathrm{C}$. The supernatant was collected, centrifuged at $10,000 \mathrm{~g}$ for $5 \mathrm{~min}$ to remove any detached cells and debris, and used immediately as a potential stimulus for isolated, previously unstimulated neutrophils.

Adherence assay under static conditions. A visual adherence assay was utilized as previously described (6). It should be noted that the procedure used in this study does not utilize shear stress as with techniques requiring a washing step $(4,5,10,35-37)$. HUVEC monolayers on $25-\mathrm{mm}$ round glass coverslips were washed by dipping three times in two changes of PBS and immediately inserted into the adherence chambers specifically made for use with an inverted microscope and phase-contrast optics. The chambers consisted of two metal plates designed to hold two $25-\mathrm{mm}$ round cover glasses separated by a SykesMoore chamber O-ring (Bellco Glass Inc., Vineland, NJ). Within this closed compartment neutrophils could be observed as they contacted the HUVEC monolayer. Neutrophils suspended in PBS $\left(10^{6}\right.$ cells $\left./ \mathrm{ml}\right)$ were injected into the chamber and allowed to settle onto the monolayer for a period of $500 \mathrm{~s}$. The number of neutrophils in contact with the monolayer was determined by counting at least 10 microscopic fields ( $50 \times$ objective), and the chamber inverted for an additional 500 s. The percentage of cells remaining in contact with the monolayer was determined and is expressed in the Results as percent adherence. The percentage of cells migrating through the endothelial monolayer was also determined using previously described criteria $(6,7)$. In blocking experiments, neutrophils were pretreated for $5 \mathrm{~min}$; then the cell suspension was injected into the adherence chamber without washing. This adhesion assay was carried out at room temperature, and the migration assay was carried out at $37^{\circ} \mathrm{C}$ to increase the percentage of migrating cells. Chemotactic stimulation of neutrophils prior to assessing adhesiveness was performed in the following manner: Neutrophils suspended in PBS $\left(10^{7}\right.$ cells $\left./ \mathrm{ml}\right)$ were exposed to $10 \mathrm{nM}$ FMLP for 30 min at $37^{\circ} \mathrm{C}$, then diluted to $10^{6} / \mathrm{ml}$ in PBS immediately before being injected into the adhesion chamber.

Adherence assay under conditions of flow. A modification of a previously published procedure $(8,15)$ was used. Passage one HUVEC were grown to confluence on fibronectin-coated coverslips (Bellco Glass, Inc.), stimulated with IL-1 $(5 \mathrm{U} / \mathrm{ml})$ for 3-4 h, rinsed in serumfree medium (RPMI1640) and mounted in parallel plate flow chambers. Neutrophils were suspended in culture medium (RPMI1640) without serum, and treated as described in the text for each specific experiment. The neutrophil suspensions were passed through the chamber at a wall shear stress of 0.36 or $1.85 \mathrm{dyn} / \mathrm{cm}^{2}$, and 
the interaction of neutrophils with the endothelial monolayer was observed under phase-contrast microscopy (Diaphot-TMD, Nikon, Inc., Garden City, NY) for $10 \mathrm{~min}$ and recorded on video tape. Digitized frames (Perspective System, Inc., Houston, TX) from videotaped experiments were used to determine the number of neutrophils that remained in contact with the monolayer. Transendothelial migration was evaluated as previously described (8) using essentially the same criteria as in the static assay. All experiments using the flow chamber were carried out at $37^{\circ} \mathrm{C}$.

Data presentation. Results are presented as means $\pm 1 \mathrm{SD}$, and $n$ is the number of separate experiments. Statistical assessments were made using analysis of variance and Dunnett's $t$ test, or Student's $t$ test.

\section{Results}

Chemotactic stimulation inversely regulates the functional activity of CD11/CD18 and neutrophil LECAM-1. As shown previously (30), antibodies recognizing CD18 and neutrophil LECAM-1 bound to most neutrophils. The loss of binding of Leu8 and increased binding of R15.7 after chemotactic stimulation for $30 \mathrm{~min}$ at $37^{\circ} \mathrm{C}$ involves most of the neutrophils (Fig. 1). In an effort to assess the rate at which the LEC-CAM was lost from the neutrophil surface after stimulation, viable neutrophils were directly sampled for flow cytometry at 1-min intervals after addition of $10 \mathrm{nM}$ FMLP (Fig. 2). A significant rise in Leu 8 binding was seen at $1 \mathrm{~min}$ after stimulation followed by a significant drop in binding that was evident at $3 \mathrm{~min}$ and almost complete by $10 \mathrm{~min}$. Unstimulated cells failed to show either a rise or fall in Leu8 binding over this observation period (data not shown). As shown in Fig. 3, CD18-deficient neutrophils exhibited less binding of DREG-56 than normal cells $(P$ $<0.01, n=5$ ), although after exposure to FMLP for $15 \mathrm{~min}$ at $37^{\circ} \mathrm{C}$, MAb binding was reduced to the same low level as that of stimulated normal cells. Neither immunofluorescence microscopy nor flow cytometry detected binding of DREG-56 to unstimulated or IL-1-stimulated endothelial cells.
We have also shown that DREG-56 significantly reduces the adhesion of unstimulated adult and CD18-deficient neutrophils to IL-1-stimulated HUVEC (23a), and that chemotactic stimulation under conditions shown to reduce markedly the amount of surface antigen recognized by DREG-56 significantly reduces adhesion to IL-1-stimulated HUVEC. The data in Fig. 4 confirm and extend these observations by indicating the following: (a) DREG-56 significantly inhibited the adhesion of neutrophils. $(b)$ The inhibition of adhesion of normal neutrophils in the presence of a combination of DREG-56 and either R15.7 or R3.1 was significantly greater than the inhibition caused by either MAb alone. (c) The binding of two different antibodies to the neutrophil surface did not enhance the inhibitory activity of either DREG-56 or R 15.7. (d) Chemotactic stimulation under conditions shown above to markedly reduce the binding of DREG-56 to the neutrophil significantly reduced the adhesion of neutrophils exposed to $R$ 15.7. These data also indicate that FMLP stimulation and its associated loss of the surface antigen recognized by Leu8 and DREG-56 reduced adhesion of CD18-blocked cells to a greater degree than DREG-56. Previous studies have shown that CD18-deficient neutrophils adhere to IL-1 stimulated HUVEC monolayers at $\sim 50 \%$ the level of normal neutrophils (7). Consistent with our previous studies (23a), DREG-56 reduced adhesion of CD18-deficient neutrophils by $38.4 \pm 5 \%(n=3, P<0.01)$, and additionally, preincubation for $30 \mathrm{~min}$ at $37^{\circ} \mathrm{C}$ in $10 \mathrm{nM}$ FMLP (thereby reducing DREG-56 binding from a mean fluorescent channel of $105 \pm 17$ to $12 \pm 3$ ) reduced adhesion by $65.4 \pm 4 \%(P<0.01, n=3)$.

The basal level of adherence of normal neutrophils to unstimulated HUVEC $(14.7 \pm 1.6 \%, n=3)$ was not significantly influenced by incubation with $50 \mu \mathrm{g} / \mathrm{ml}$ DREG-56 $(17.8 \pm 1.8 \%, n=3)$, but was significantly reduced in the presence of $5 \mu \mathrm{g} / \mathrm{ml} \mathrm{R} 15.7(3.1 \pm 2.0 \%, n=3, P<0.01)$. These results indicate that the endothelial structure with which neu-
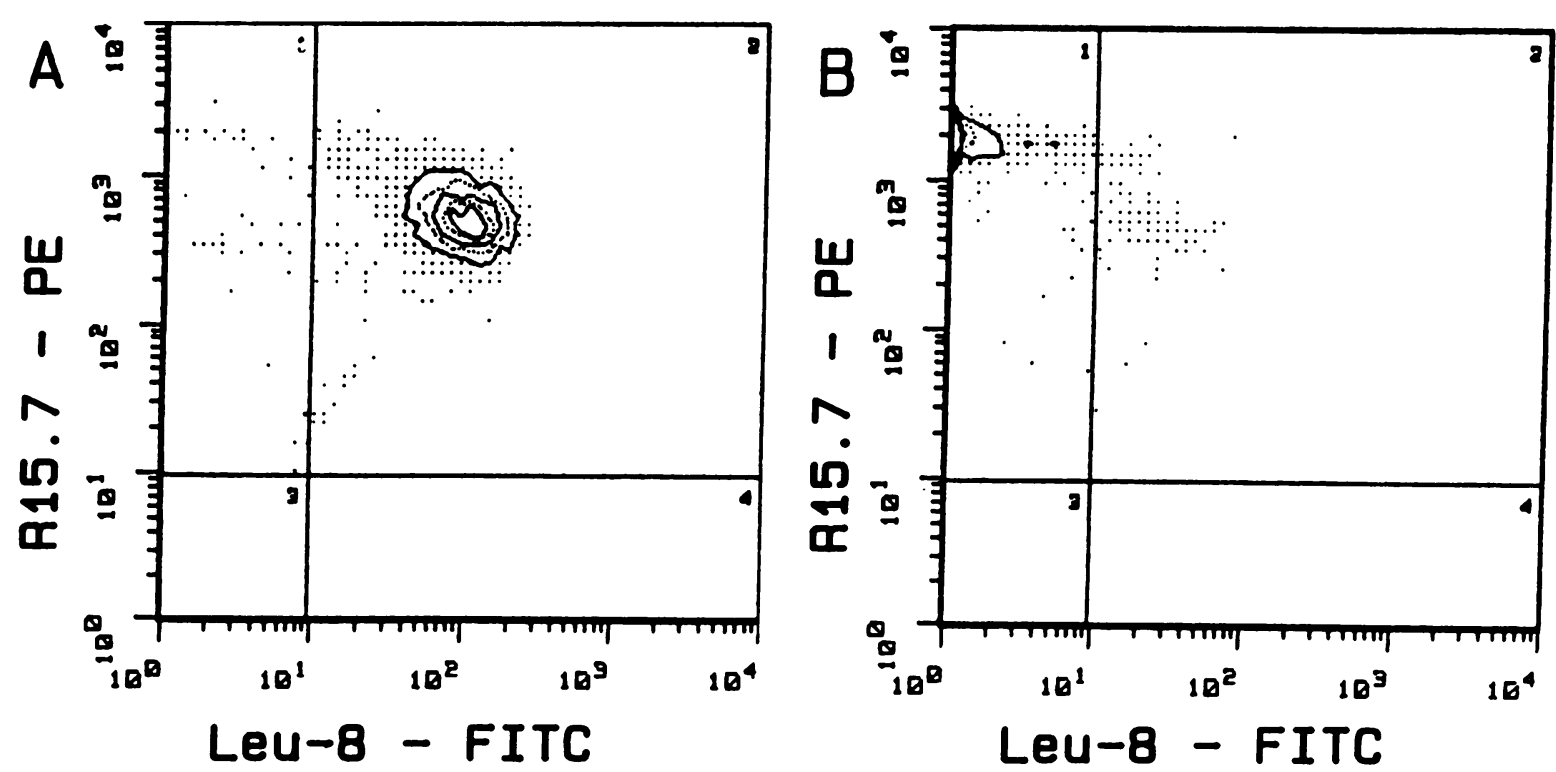

Figure 1. Effect of chemotactic stimulation on the binding of R15.7 and Leu8 to normal human neutrophils. Isolated normal neutrophils were incubated at $37^{\circ} \mathrm{C}$ for $30 \mathrm{~min}$ in PBS $(A)$ or PBS containing $10 \mathrm{nM}$ FMLP $(B)$, and washed twice in PBS. Biotin-labeled R15.7 and FITC-labeled Leu8 were both added to the cell suspension, and PE-labeled avidin was used to detect the R15.7. The quadrant markers were set using neutrophils exposed to nonbinding isotype matched PE- and FITC-labeled MAbs. Fluorescence channels are plotted on a log scale: PE on the $x$ axis and FITC on the $y$ axis. Similar results were obtained using cells from four donors. 


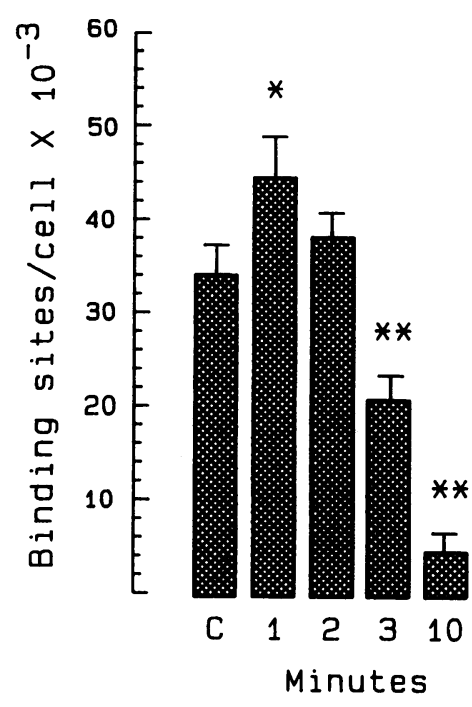

trophil LECAM-1 interacts is not present on unstimulated endothelial cells.

Neutrophil adhesion to endothelial cells under shear forces involves LECAM-1 on the neutrophil surface but not CD18. In previously published studies, we have evaluated the adhesion of neutrophils to HUVEC monolayers under conditions of flow, and have shown that at wall shear stresses between 1 and $2 \mathrm{dyn} / \mathrm{cm}^{2}$, the adhesion of unstimulated neutrophils is markedly enhanced by stimulating HUVEC with IL- $1(2 \mathrm{U} / \mathrm{ml}$, $\left.37^{\circ} \mathrm{C}\right)$ for $3 \mathrm{~h}(8,15)$. The adhesion at $2.0 \mathrm{dyn} / \mathrm{cm}^{2}$ was not inhibited by MAbs TS1/18 (anti-CD18) or R6.5 (anti-ICAM1). Moreover, cells from one patient with CD18-deficiency were shown to adhere normally under these experimental conditions $(8,9)$. In the present studies with DREG-56, adhesion was evaluated at a wall shear stress of $1.85 \mathrm{dyn} / \mathrm{cm}^{2}$. The data revealed that as previously shown, adhesion was not significantly reduced by addition of anti-CD18 MAb, TS1/18 (percent inhibition, 14.3 $\pm 14.1, n=4)$. However, adhesion at this wall shear stress was significantly reduced by DREG-56 (Fig. 5). The effect of this MAb was greater than that revealed by the

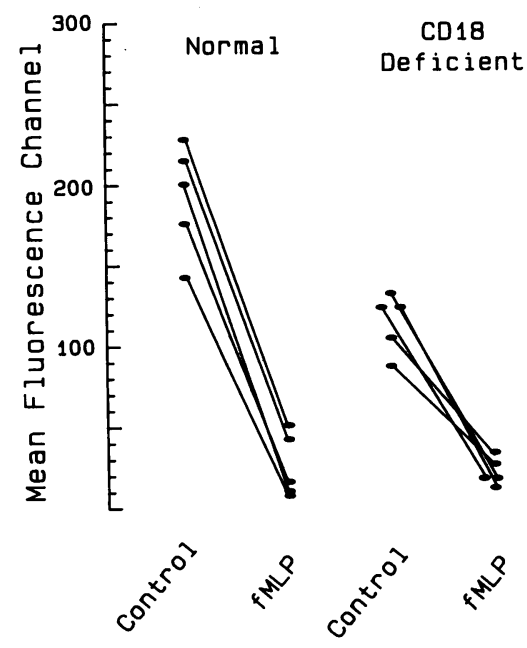

Figure 3. Effect of chemotactic stimulation on the binding of DREG-56 to normal and CD18-deficient neutrophils. Neutrophils were incubated at $37^{\circ} \mathrm{C}$ for 30 min in PBS or PBS containing $10 \mathrm{nM}$ FMLP, washed, and exposed to DREG-56 for $15 \mathrm{~min}$. Binding of DREG-56 was detected using FITC-labeled goat anti-mouse IgG. The mean values for unstimulated normal and CD18-deficient cells were significantly different $(P<0.01)$. After stimulation, the values were not different. assay performed without shear stress, and approached that seen under less regulated shear conditions at $7^{\circ} \mathrm{C}$ (23a). Preincubation of neutrophils with FMLP under conditions that produced marked reduction in the surface antigen recognized by DREG56 (i.e., $10 \mathrm{nM}, 30 \mathrm{~min}, 37^{\circ} \mathrm{C}$ ) significantly diminished their adhesion. In similar experiments with CD18-deficient neutrophils from three patients, DREG-56 reduced adhesion by $47.4 \pm 9.8 \%(P<0.01, n=4)$, and chemotactic stimulation reduced adhesion by $66.1 \pm 5.9 \%(n=2)$. Also evident in these studies was the finding that neither removal of LECAM-1 from the neutrophil surface nor blocking with antibodies to LECAM-1 completely prevented adhesion under conditions of flow.

Studies performed at a wall shear stress of $0.36 \mathrm{dyn} / \mathrm{cm}^{2}$, a condition where CD18 plays some adhesive role, revealed an additive effect of both TS1/18 (anti-CD18) and DREG-56. DREG-56 inhibited adhesion by $30.3 \pm 10.2 \%$, TS1/18 inhibited by $25.4 \pm 12.5 \%$, and when both MAb were present, inhibition was $50.9 \pm 8.6 \%(P<0.05$ compared with either MAb alone, $n=5$ ).

Additional experiments were performed to assess the requirement for neutrophil LECAM-1 in adhesion under conditions of flow. As shown in Fig. 6, neutrophils stimulated with various concentrations of FMLP exhibited partial reductions in surface LECAM-1 commensurate with the level of stimulation, and adhesion at $1.85 \mathrm{dyn} / \mathrm{cm}^{2}$ was highly correlated with the neutrophil surface level of LECAM-1.

Stimulated endothelial cells induce down-regulation of neutrophil LECAM-1. Kishimoto et al. (29) presented the hypothesis that in the mouse, the MEL-14 antigen is responsible for the initial adhesive interactions between neutrophils and endothelial cells, with subsequent involvement of CD18-dependent adhesion as neutrophils are activated in situ and migrate through the endothelium. Detachment of the adhesive mechanism dependent on neutrophil LECAM-1 is an essential event in this scheme. From the results presented above, it appears that LECAM-1 may play such a carefully coordinated role in human neutrophil-endothelial adhesion. In order to assess whether detachment of this adhesive mechanism occurs, the following experiments were performed.

Using the static adhesion assay at $37^{\circ} \mathrm{C}$, previously unstimulated neutrophils were allowed to contact IL-1-stimulated $\left(3 \mathrm{U} / \mathrm{ml}, 3 \mathrm{~h}, 37^{\circ} \mathrm{C}\right)$ endothelial monolayers for $500 \mathrm{~s}$. The monolayers were inverted for an additional $500 \mathrm{~s}$, and the percent adherent cells determined. In order to prevent CD18-dependent migration (7), R15.7 (anti-CD18) was included with normal neutrophils $(5 \mu \mathrm{g} / \mathrm{ml})$, or CD18-deficient neutrophils were used. Incubation of the chambers was continued in the inverted position for up to an hour. The level of neutrophil adhesion was determined at 20,30 , and $60 \mathrm{~min}$. Normal cells treated with R15.7 and CD18-deficient neutrophils detached at the same rate (Fig. 7), and reached a low level of adhesion within $30 \mathrm{~min}$. The detached cells were flushed from the chamber and evaluated for the ability to bind FITC-labeled Leu8. Compared with neutrophils not in contact with the HUVEC monolayer, binding of this MAb was markedly reduced (mean fluorescent channel of control cells $86 \pm 5, n=3$; neutrophils from chambers, $18 \pm 4, n=3$ ). In contrast, $>90 \%$ of normal cells without R15.7 remained associated with the HUVEC over this observation period, and as previously shown $(6,7)$, most of the cells rapidly migrated between the monolayer and the substratum. 


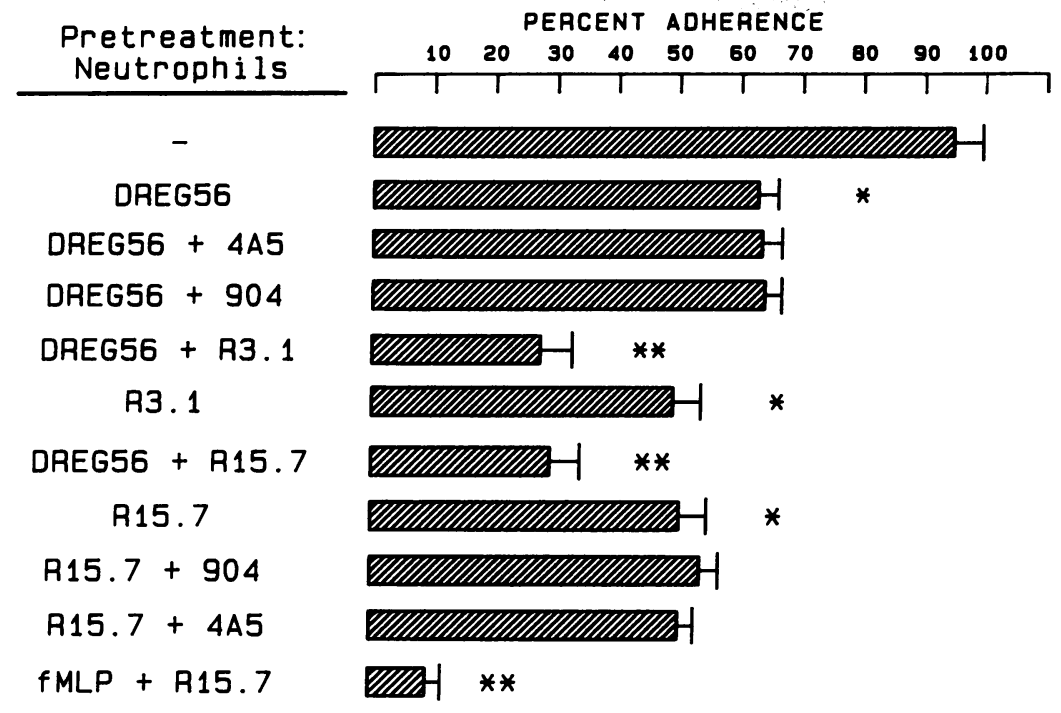

Figure 4. Adhesion of neutrophils to IL-1-stimulated HUVEC monolayers: effects of MAbs and chemotactic stimulation. HUVEC monolayers were stimulated with IL-1 for $4 \mathrm{~h}$, washed, and inserted into adhesion chambers. Isolated neutrophils were suspended in PBS with and without MAbs as indicated $(10 \mu \mathrm{g} / \mathrm{ml})$, or incubated in PBS containing $10 \mathrm{nM}$ FMLP for $30 \mathrm{~min}$ before being suspended in PBS containing R15.7. Neutrophil suspensions containing antibodies were injected into the chambers and adhesion was assessed in the absence of shear stress. The MAbs used were 904 (anti-CD1 1b), R15.7 (anti-CD18), R3.1 (anti-CD11a), DREG-56 (antibody recognizing the human homologue of the murine MEL-14 antigen), and 4A5 (anti-human neutrophil, unknown antigen). Values shown are mean \pm 1 SD for four separate experiments. ${ }^{*} P<0.01$ compared with untreated cells; ${ }^{* *} P<0.01$ compared with cells treated only with R15.7 or R3.1.
In order to compare the effect of neutrophil contact with unstimulated and IL-1 stimulated endothelial cells, neutrophils were allowed to remain on HUVEC monolayers for 30 min or $1 \mathrm{~h}$ at $37^{\circ} \mathrm{C}$, collected by rinsing the monolayers with PBS, and evaluated for binding of FITC labeled Leu8. Both R15.7-treated normal neutrophils and CD18-deficient neutrophils on unstimulated HUVEC exhibited the same level of binding as controls, i.e., cells not in contact with the monolayers and maintained at $4^{\circ} \mathrm{C}$ in $\mathrm{PBS}$ or incubated at $37^{\circ} \mathrm{C}$ with IL-1 in PBS. In contrast, neutrophils on IL-1-stimulated HUVEC had greatly reduced binding of Leu8 (Fig. 8).

The possibility that a soluble factor was released from stimulated HUVEC monolayers was evaluated by collecting supernatants from both unstimulated and stimulated monolayers. Neutrophils were exposed to these supernatants and evaluated for the ability to bind DREG-56. Supernatants from unstimulated HUVEC monolayers were without effect, while those from IL-1-stimulated HUVEC produced marked reductions in the neutrophil's ability to bind DREG-56 (Fig. 9), and to adhere to IL-1-stimulated HUVEC monolayers (Table I). Flow cytometric analysis revealed that most neutrophils had increased surface Mac-1 (as shown by binding of Leu-15) and decreased binding of DREG-56. Additionally, the time course over which these modulations in surface antigens occurred following exposure to either culture supernatant or FMLP (Fig. 10) was consistent with the rate of detachment of neutrophils from IL-1-stimulated HUVEC monolayers (Fig. 7).

PAF and the endothelial form of interleukin 8 (IL-8) (3840) are produced by cytokine-stimulated endothelial cells. Both are chemotactic for neutrophils and as shown in Fig. 11, both can induce the coincident up-regulation of Mac-1, and loss of LECAM-1 from the neutrophil surface.

Anti-CD18 but not DREG-56 blocks neutrophil transendothelial migration. IL-1-stimulated HUVEC promote transendothelial migration of previously unstimulated normal neutrophils $(6,41,42)$. This appears to be largely a CD18-dependent process since anti-CD18 MAbs produce $>90 \%$ inhibition, and CD18-deficient neutrophils exhibit $<3 \%$ of the migration of normal cells (6). The possibility that the antigen recognized by DREG-56 also plays a role in this migration was investigated by quantitating migration in the presence of this MAb. In the assay performed without shear stress, a concentration of DREG-56 sufficient to produce maximum reduction in adhesion $(50 \mu \mathrm{g} / \mathrm{ml})$ did not reduce the number of migrating neutrophils (Fig. 12), while in parallel studies, R15.7 almost completely inhibited migration, 904 (anti-CD11b) produced

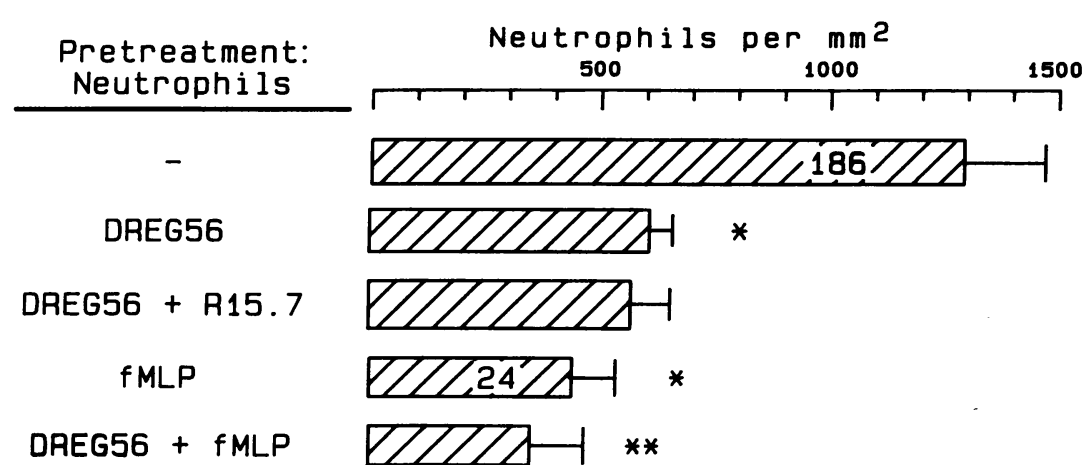

Figure 5. Adhesion of neutrophils to IL-1stimulated HUVEC monolayers under conditions of flow: effects of monoclonal antibodies. HUVEC monolayers were stimulated with IL-1 for $4 \mathrm{~h}$, and inserted in a parallel-plate flow chamber. Isolated neutrophils were suspended with the MAbs indicated or preincubated with FMLP (10 nM, 30 $\min , 37^{\circ} \mathrm{C}$ ), and the cell suspension was passed through the chamber at a wall shear stress of 1.85 $\mathrm{dyn} / \mathrm{cm}^{2}$. Neutrophils associated with the monolayers were enumerated (neutrophils per square millimeter of monolayer) by analysis of video tapes of the initial $10 \mathrm{~min}$ of flow made under phase-contrast microscopy. The number within the top and third bars indicates the mean fluorescent channel of flow cytometric studies of DREG-56 binding to unstimulated and FMLP-stimulated neutrophils, respectively. ${ }^{*} P<0.01$ for each experimental condition compared with untreated control cells; ${ }^{* *} P<0.05$ compared with DREG-56 alone. $n=5$. 


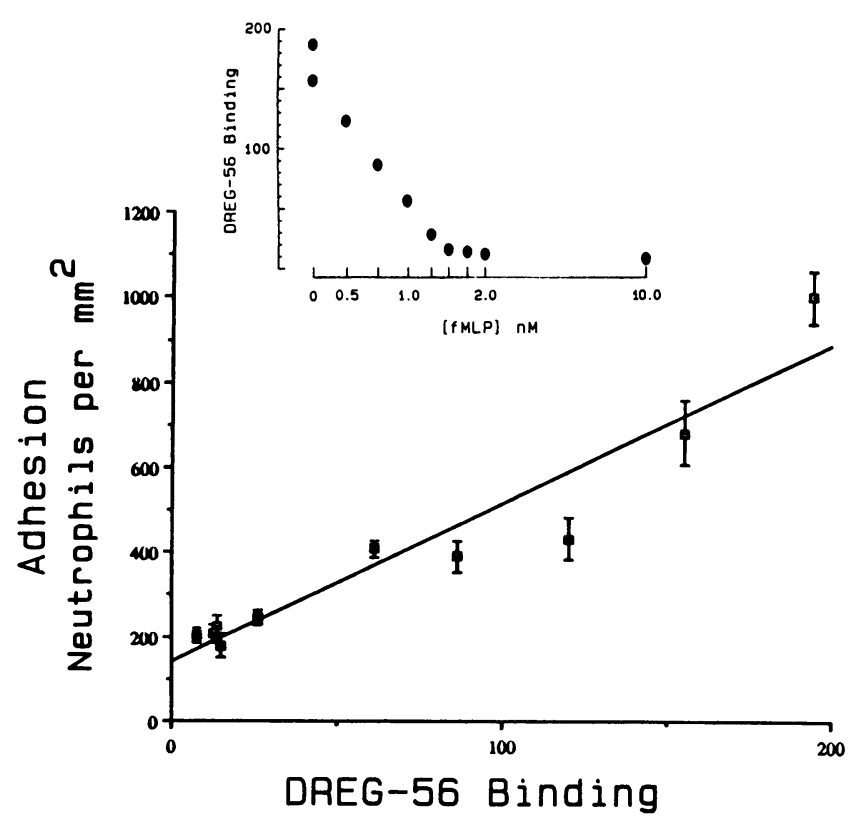

Figure 6. Adhesion of neutrophils to IL-1-stimulated HUVEC monolayers under conditions of flow: effects of chemotactic stimulation. HUVEC monolayers were stimulated with IL-1 for $4 \mathrm{~h}$, and inserted in a parallel-plate flow chamber. Isolated neutrophils were preincubated with various concentrations of FMLP (30 min, $37^{\circ} \mathrm{C}$ ) as shown in the inset graph, washed, and resuspended in medium, and the cell suspension was passed through the chamber at a wall shear stress of $1.85 \mathrm{dyn} / \mathrm{cm}^{2}$. Neutrophils associated with the monolayers were enumerated (neutrophils per square millimeter of monolayer) by analysis of video tapes of the initial $10 \mathrm{~min}$ of flow made under phase-contrast microscopy.

partial inhibition, and a control MAb (4A5) that binds to neutrophils without inhibiting adhesion (7) failed to influence migration. In the assay performed under flow, the proportion of adherent neutrophils that migrated beneath the monolayer in the presence of DREG-56 $(70.6 \pm 8.1 \%, n=10)$ was the same as in the absence of MAbs $(68.6 \pm 8.9 \%, n=16)$. However, the total number of migrated cells per square millimeter was reduced by DREG-56 since this antibody significantly reduced the number of neutrophils adhering to the endothelium and thereby the number of cells available to migrate (migrated cells per square millimeter without MAbs, $697 \pm 171, n=16$; with DREG-56, 354 $\pm 77, n=10, P<0.01)$.

\section{Discussion}

The current studies demonstrate that CD18 integrins, Mac-1 and LFA-1, and LECAM-1 on the human neutrophil surface play distinct roles in the adhesion of these leukocytes to human endothelial cells in vitro. The distinctions can be clearly identified in three areas: $(a)$ neutrophil-endothelial adhesion under conditions of flow, $(b)$ adhesion after chemotactic stimulation lation of the neutrophils, and $(c)$ transendothelial migration induced by cytokine stimulation of the endothelial cells.

Human neutrophils will adhere to IL-1-stimulated HUVEC monolayers in vitro in parallel plate flow chambers at a wall shear stress of $\sim 2.0 \mathrm{dyn} / \mathrm{cm}^{2}(15)$. Monoclonal antibod- ies against CD18 that markedly inhibit adhesion to IL-1-stimulated HUVEC under static conditions, are ineffective in reducing adhesion at wall shear stresses in this range in vitro $(8,9)$. In contrast, DREG-56 is more effective in reducing adhesion under these flow conditions than under static conditions, indicating that LECAM-1 on the surface of neutrophils may play a significant role in neutrophil-endothelial adherence under conditions where leukocyte integrins are ineffective. This concept is supported by three additional observations. The first is that neutrophils from patients with $\mathrm{CD} 18$ deficiency exhibit high levels of adhesion to IL-1-stimulated HUVEC at wall shear stresses $\sim 2.0 \mathrm{dyn} / \mathrm{cm}^{2}(8,9)$. The second is that chemotactic stimulation of normal and CD18-deficient neutrophils under conditions shown to markedly reduce LECAM- 1 on their surface reduces adhesion under flow to same degree as DREG-56, and combination of chemotactic pretreatment and DREG-56 produces no further reduction in adherence. The third is that the level of LECAM-1 on the neutrophil surface is directly related to the capacity to adhere to IL-1-stimulated HUVEC under conditions of flow.

Chemotactic stimulation of normal neutrophils increases their adhesion to endothelial cells under static conditions (1-4, $7,10)$ or flow with very low wall shear stresses $\left(0.25 \mathrm{dyn} / \mathrm{cm}^{2}\right)$ in vitro $(8,9)$. This effect appears to be primarily dependent on Mac-1 $(4,5,7,10)$, as shown by the fact that MAbs against $\mathrm{CD} 11 \mathrm{~b}$ and $\mathrm{CD} 18$ reduce adhesion to near unstimulated levels. The observation that chemotactic stimulation removes the majority of LECAM-1 from the neutrophil surface seems to preclude a role for LECAM-1 in the adhesion of chemotactically stimulated neutrophils, a conclusion supported by the results of two experiments. Adhesion of CD18-deficient neutrophils to IL-1-stimulated HUVEC under static conditions was signifi-

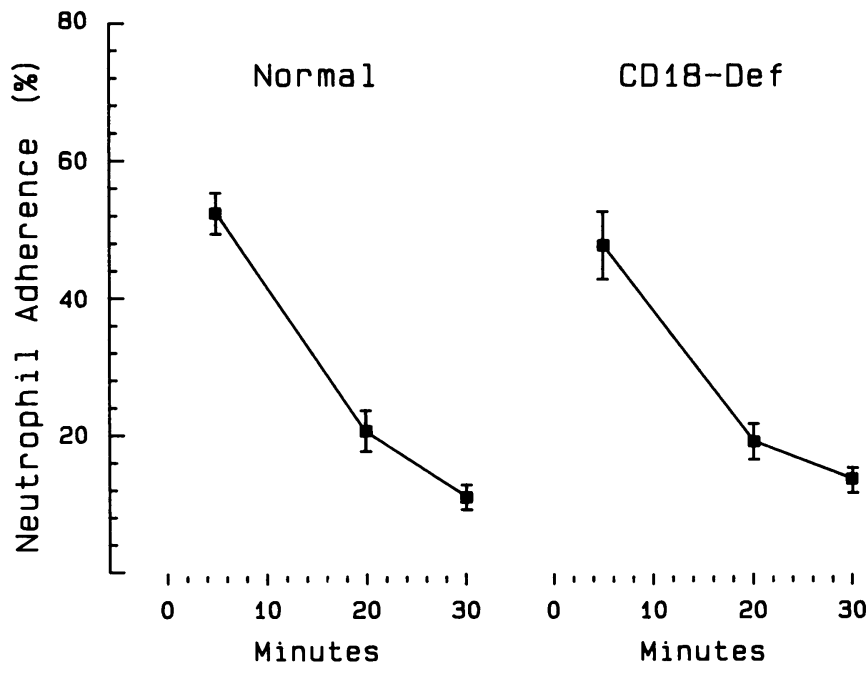

Figure 7. Detachment of neutrophils from IL-1-stimulated HUVEC monolayers. HUVEC monolayers were stimulated for $3 \mathrm{~h}$ with IL-1, rinsed in PBS, and inserted into adherence chambers. Control neutrophils were suspended in PBS containing $10 \mu \mathrm{g} / \mathrm{ml} \mathrm{R} 15.7$, and CD18-deficient neutrophils were suspended in PBS before being injected into the chamber. Neutrophils were allowed to settle onto the monolayer for $500 \mathrm{~s}$, the chamber was inverted for $500 \mathrm{~s}$, and the percentage of adherent cells was determined immediately and at 20 and $30 \mathrm{~min}$. Number of separate experiments, six using normal cells, and three using CD18-deficient cells. 


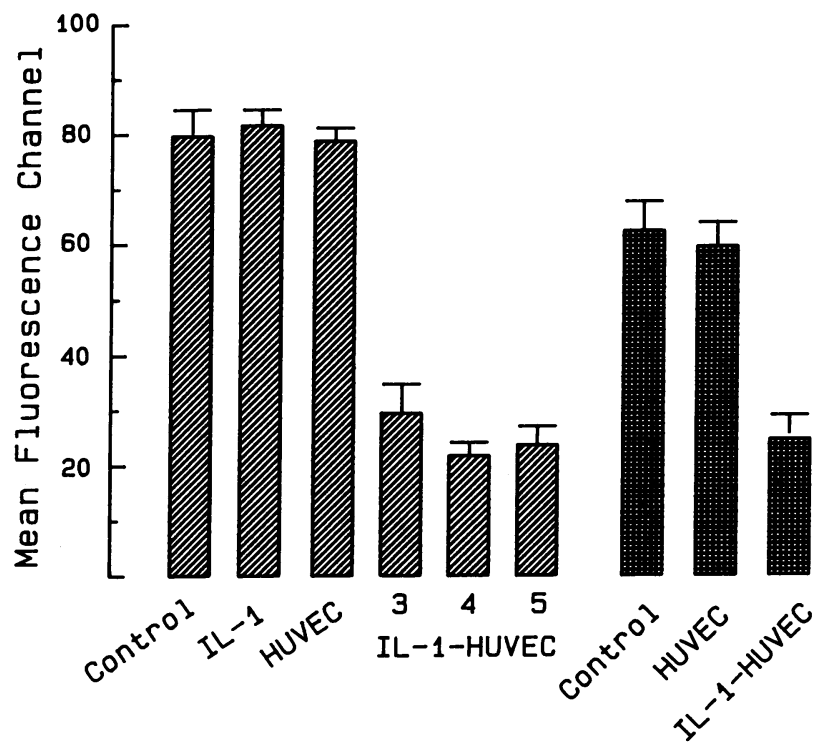

Figure 8. Effect of neutrophil-HUVEC monolayer contact on FITC labeled Leu 8 binding to neutrophils. HUVEC monolayers were incubated at $37^{\circ} \mathrm{C}$ for 3,4 , or $5 \mathrm{~h}$ as indicated with IL- 1 or left unstimulated (bars labeled HUVEC), rinsed, drained, and placed in a plastic culture dish. A suspension of neutrophils $\left(10^{6} \mathrm{in} 0.3 \mathrm{ml}\right)$ was placed on the monolayer, and incubated at $37^{\circ} \mathrm{C}$ for $30 \mathrm{~min}$. Then the neutrophils were collected by rinsing the monolayer with $1 \mathrm{ml}$ of PBS, and evaluated for Leu8 binding using flow cytometry. Control neutrophils were maintained in suspension without stimulation or in the presence of IL-1 $(5 \mathrm{U} / \mathrm{ml})$ for $30 \mathrm{~min}$ before assessing the binding of Leu8. Number of separate experiments, six using normal cells (hatched bars), and three using CD18-deficient cells ( fine screen bars).

cantly reduced by chemotactic stimulation, and adhesion of normal neutrophils under static conditions was reduced to very low levels by a combination of chemotactic stimulation and treatment with anti-CD18 MAb.

A striking feature of cytokine-stimulated HUVEC monolayers is that they induce transendothelial migration of adherent human neutrophils $(6,41,42)$. Though the endothelial factor(s) responsible for stimulating this neutrophil motility have not been defined, the adhesion molecules on the neutrophil involved in this event appear to be primarily leukocyte integrins, specifically Mac-1 and LFA-1 $(7,9)$. This conclusion is supported by the following findings: (a) MAbs against the $\alpha$ subunits of these integrins are capable of decreasing migration by up to $50 \%$ when used alone and by greater that $90 \%$ when added together; $(b)$ transendothelial migration of CD18-deficient cells is very infrequent; and $(c)$ DREG-56 does not inhibit migration of adherent normal neutrophils. This conclusion is also reasonable in light of the observation that chemotactic stimulation induces a loss of LECAM-1 from the neutrophil surface. If the endothelial-dependent leukocyte migration results from chemotactic factors produced by the monolayer, such stimulation would likely result in the loss of LECAM-1. Results in this report directly show that endothelial derived factors stimulate a rapid loss of LECAM-1 from the neutrophil, and recent studies show that neutrophil migration through HUVEC monolayers toward chemotactic gradients is almost completely inhibited by anti-CD18 MAbs and unaffected by

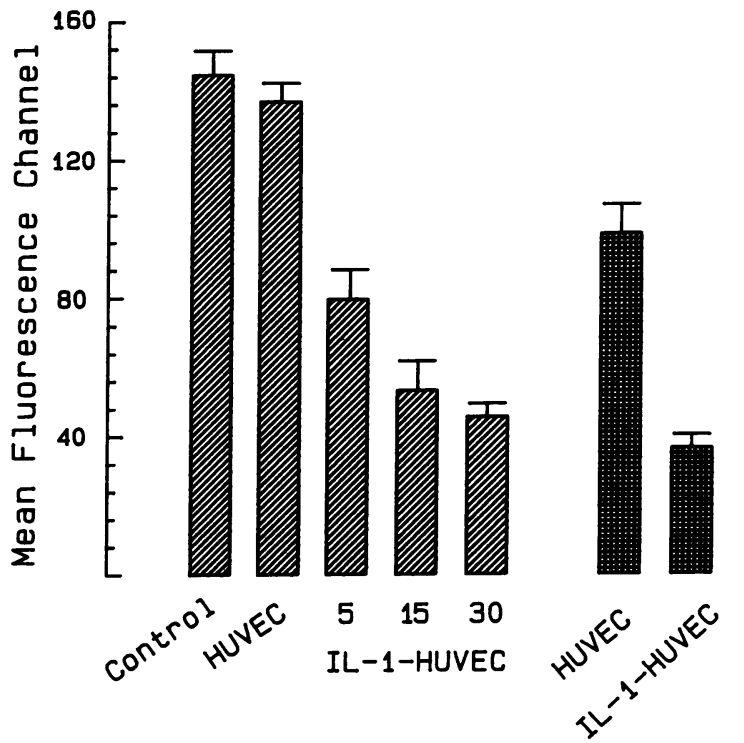

Figure 9. Effect of supernatant from IL-1-stimulated HUVEC on the binding of DREG-56 to neutrophils. HUVEC monolayers on 25-mm round coverglasses were stimulated at $37^{\circ} \mathrm{C}$ for $3 \mathrm{~h}$ with IL-1. The culture medium was removed from these and control monolayers (bars labeled HUNEC), and the monolayer covered with $0.3 \mathrm{ml}$ Dulbecco's complete balanced salt solution with glucose for an additional hour. This conditioned supernatant was collected, and centrifuged to remove any detached endothelial cells. Neutrophils were collected by centrifugation, resuspended in this supernatant for 5,15 , or $30 \mathrm{~min}$, and then evaluated for the binding of DREG-56 using flow cytometry. Control neutrophils were held in suspension without stimulation for $30 \mathrm{~min}$ before assessing binding of DREG-56. Number of separate experiments, six using normal cells (hatched bars) and three using CD18-deficient cells (fine screen bars).

DREG-56 (Furie, M. B., M. C. A. Tancinco, and C. W. Smith, manuscript submitted for publication).

Kishimoto et al. (29) put forward an hypothesis that murine

Table I. Effects of HUVEC Culture Supernatant on Neutrophil Adhesion to IL-1-stimulated HUVEC

\begin{tabular}{|c|c|c|}
\hline $\begin{array}{c}\text { Pretreatment of } \\
\text { neutrophils* }\end{array}$ & $\begin{array}{c}\text { Percent } \\
\text { adherence }\end{array}$ & Leu8 \\
\hline & \multicolumn{2}{|c|}{$\%$} \\
\hline PBS & $51 \pm 12$ & $87 \pm 10$ \\
\hline FMLP, $10 \mathrm{nM}$ & $9 \pm 1^{\ddagger}$ & $5 \pm 3^{\ddagger}$ \\
\hline HUVEC & $55 \pm 11$ & $83 \pm 9$ \\
\hline IL-1 HUVEC' & $18 \pm 4^{\ddagger}$ & $33 \pm 8^{\ddagger}$ \\
\hline
\end{tabular}

* Neutrophils were incubated in PBS containing the indicated stimulant for $30 \mathrm{~min}$ at $37^{\circ} \mathrm{C}$, then divided into two aliquots. One was analyzed by flow cytometry, and the other was suspended in PBS containing a final concentration of R15.7 of $10 \mu \mathrm{g} / \mathrm{ml}$ and used for adherence determinations. Adherence was determined as a percentage of cells contacting the monolayer in the static assay, and Leu-8-FITC binding was determined and expressed as mean fluorescence channel. ${ }^{\ddagger} P<0.01, n=3$. Results expressed as means $\pm \mathrm{SD}$, from duplicate determinations in three separate experiments.

Supernatant collected from unstimulated HUVEC monolayers without further dilution or concentration.

I Supernatant collected from IL-1-stimulated HUVEC during the 4th $h$ after addition of IL-1, $3 \mathrm{U} / \mathrm{ml}, 37^{\circ} \mathrm{C}$. 

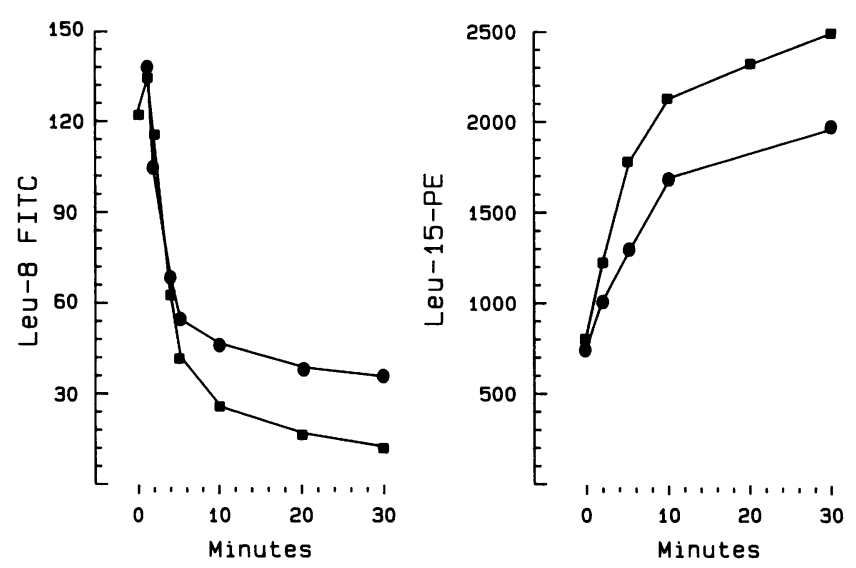

Figure 10. Binding of Leu-8 and Leu-15 to the surface of normal neutrophils: changes induced by incubation with FMLP- or HUVEC-conditioned PBS. Neutrophils suspended with Leu8-FITC and Leu 15-PE were incubated at $37^{\circ} \mathrm{C}$ with FMLP $(10 \mathrm{nM})$ or supernatant from IL-1-stimulated HUVEC monolayers (circles) previously determined to stimulate neutrophils. The suspension was repeatedly sampled for flow cytometry. 2,000 cells were analyzed at each time indicated after introduction of the stimulant. These results are representative of three separate experiments.

neutrophil adhesion to endothelial cells under inflammatory conditions involves at least two distinct sets of adhesive mechanisms. One involves LECAM-1 on the neutrophil surface, and the second involves leukocyte integrins. Two key features of this hypothesis are first, that these mechanisms act sequentially in vivo, the LECAM-1 mechanism being responsible, at least in part, for the initial adhesion, and leukocyte integrins being responsible for continued adhesion and subsequent emigration, and second, that the initial mechanism is "down-regulated" as the second mechanism is engaged, presumably by chemotactic factors in the inflammatory site. Additional evidence supporting this hypothesis and its application to human cells comes from the results in this and a separate report (23a). Two experiments have direct bearing on the first point. As discussed above, neutrophils flowing by an IL-1-stimulated HUVEC monolayer can adhere by a mechanism dependent on the neutrophil surface LECAM-1, and transendothelial migration is primarily a CD18-dependent event. Two experiments have direct bearing on the second feature. CD18-deficient neutrophils in contact with IL-1-stimulated, but not unstimulated HUVEC monolayers exhibited a rapid loss of ability to bind DREG-56 or Leu8, and coincident detachment from the monolayer. Detachment and loss of LECAM-1 were demonstrated with normal neutrophils when CD18-dependent adhesion and migration were blocked by addition of anti-CD18 MAb, thus allowing recovery of the cells in contact with HUVEC. The time course of this event was similar to that when chemotactic factor was added to cells in suspension. Supernatant from IL1-stimulated, but not unstimulated HUVEC monolayers induced a rapid loss of LECAM-1 from the neutrophil surface associated with a significantly reduced ability to adhere to stimulated endothelial cells. These observations provide direct evidence for the sequential operation of these distinct adhesive mechanisms.

The identity of the endothelial surface determinant interacting with neutrophil LECAM-1 is unknown. Since the adhe-

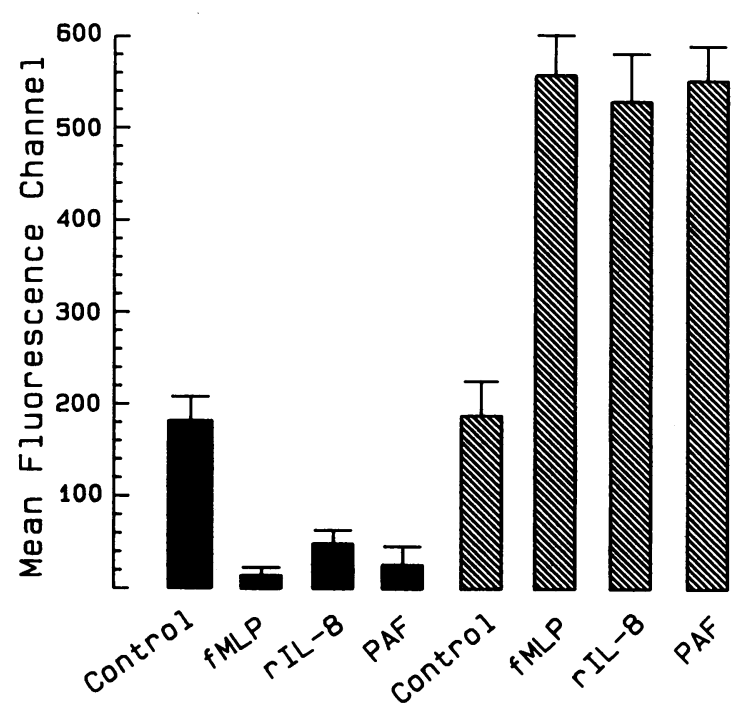

Figure 11. Effects of PAF and endothelial IL-8 on binding of monoclonal antibodies to isolated neutrophils. Neutrophils were suspended in PBS alone or PBS containing FMLP (10 nM), IL-8 (200 $\mathrm{ng} / \mathrm{ml})$, or PAF $(10 \mathrm{ng} / \mathrm{ml})$ for $30 \mathrm{~min}$ at $37^{\circ} \mathrm{C}$, and then prepared for flow cytometry using MAbs DREG-56 (anti-neutrophil LECCAM, solid bars) or R15.7 (anti-CD18, hatched bars). Mean fluorescent channel $( \pm \mathrm{SD})$ for four separate experiments is plotted.

sion dependent on LECAM- 1 is demonstrable only after IL-1 stimulation of the HUVEC monolayer, the determinant appears to be a newly expressed adhesive structure on the endothelial cell surface. Additional evidence for this point comes from the observations that DREG-56 does not significantly reduce the already low level of adhesion of unstimulated neutrophils on unstimulated HUVEC. It is unlikely to be ICAM-1 in light of recent experimental evidence: with regard to the adhesion of unstimulated neutrophils to IL-1-stimulated HUVEC, antiICAM-1 MAb R6.5 and DREG-56 produce additive inhibitory effects (Smith, C. W., and T. K. Kishimoto, unpublished observations), ICAM-1 is clearly a ligand for the CD18-dependent adhesion $(7,43)$, anti-ICAM-1 MAb R6.5 does not inhibit adhesion of CD18-deficient neutrophils (6), and anti-ICAM-1 does not inhibit neutrophil adhesion under flow at wall shear stresses of $2.0 \mathrm{dyn} / \mathrm{cm}^{2}(8)$.

Evidence for the possible interaction of neutrophil LECAM-1 with ELAM-1 must be considered in light of the finding that the endothelial adhesive structure appears to be newly expressed after stimulation with cytokines. In separate studies (44), we have investigated this possibility in two different experimental settings. The first involves the use of DREG-56 and anti-ELAM-1 MAbs to inhibit the adhesion of unstimulated neutrophils to IL-1-stimulated HUVEC monolayers. While anti-ELAM-1 MAbs produced additive inhibitory effects with anti-CD18 (a finding consistent with published results [18]), their effect was not additive with DREG-56. This suggests a common adhesion pathway for ELAM-1 and neutrophil LECAM-1. However, our studies also have indicated that LECAM-1 on the neutrophil surface is not the only molecule on the neutrophil surface interacting with ELAM-1, since chemotactically stimulated neutrophils having very low levels of surface LEC-CAM still exhibited some ELAM-1-dependent adhesion. In a second experimental set- 


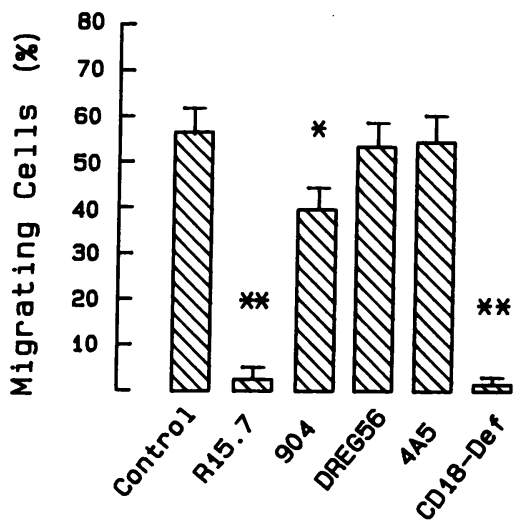

Figure 12.

Transendothelial migration of neutrophils through IL-1stimulated monolayers: effects of MAbs. HUVEC monolayers were stimulated with IL-1 for $3 \mathrm{~h}$, rinsed, and placed in adhesion chambers.

Unstimulated neutrophils in PBS or PBS containing the indicated MAbs were injected into the chambers and allowed to remain in contact with the monolayer for $20 \mathrm{~min}$. The percentage of migrating cells was then determined. Number of separate experiments was eight except for the CD18-deficient cells which was three. ${ }^{*} P<0.05$, and ${ }^{* *} P<0.01$. The labels indicate the antigens recognized by the monoclonal antibodies in that experimental condition.

ting, we found that the adhesion of unstimulated neutrophils to ELAM-1-transfected $L$ cells was partially inhibited by DREG-56. Though these preliminary results support an interaction of neutrophil LECAM-1 with ELAM-1, they also indicate that this is not an exclusive interaction. Such a finding would not be surprising if the molecular basis for the adhesive function of the LEC-CAMs is the recognition of specific carbohydrate structures (45) found on more than one surface glycoprotein. Thus, IL-1-stimulated endothelial cells promote adherence dependent on neutrophil LECAM-1 through expression of adhesive structures (possibly ELAM-1) on their surface, and they down-regulate this adhesion by releasing factor(s) that stimulate the loss of LECAM-1 from the neutrophil surface.

The identity of the endothelial-derived factor(s) that stimulate down-regulation of neutrophil LECAM-1-dependent adhesion remains to be determined. The kinetics of the cellular response, and the finding that both upregulation of Mac-1 and loss of LECAM-1 from the neutrophil surface were stimulated are consistent with a chemotactic stimulus. Three candidates are endothelial interleukin 8 (IL-8), PAF, and granulocyte/ monocyte colony-stimulating factor GM-CSF. They are produced by IL-1-stimulated HUVEC $(38-40,46)$, and as indicated in the present and other reports $(30,46)$, they can stimulate both loss of LECAM-1 from the neutrophil surface and upregulation of Mac-1. Of particular interest are the results of Gimbrone et al. (40) showing that neutrophils preincubated in endothelial-derived IL- 8 have reduced adhesion to endothelial monolayers in vitro, and the results of Peters et al. (47) showing that neutrophil localization in vivo is inhibited by systemic administration of GM-CSF. Both findings are consistent with the ability of these molecules to down-regulate the neutrophil LECAM-1 in vitro.

Studies in vivo have shown that LECAM-1 on the neutrophil surface functions as an adhesion molecule, and that there is reciprocal modulation of this molecule and CD18 integrins at sites of neutrophil localization $(27,28)$. Jutila et al. (28) found that anti-Mac-1, anti-LFA-1 and MEL-14 given intravenously in mice each produced $>60 \%$ inhibition of neutrophil accumulation in inflamed peritoneum, and that neutrophils in peritoneal exudate had low levels of surface MEL-14 antigen and high levels of surface Mac-1 when compared with neutrophils from blood or bone marrow. Kishimoto et al. (29) found that neutrophils within the lumen of blood vessels exhibited bright immunofluorescent staining for MEL-14 antigen, whereas those that had migrated into the tissues at a site of inflammation exhibited no immunofluorescent staining for MEL-14 antigen, but stained brightly for Mac-1. Thus, studies both in vitro and in vivo reveal neutrophil LECAM-1 to be an adhesion molecule functional at venous wall shear stresses, and studies in vitro indicate that the marked changes seen in surface adhesion molecules on exudate neutrophils may be due in part to factors released from inflamed endothelium.

\section{Acknowledgments}

The expert secretarial assistance of Irene Harrison is acknowledged.

This work was supported by the following grants from the National Institutes of Health: AI-23521, AI-19031, HL-42550, HL-18672, HL22236, and P50-NS-23327.

\section{References}

1. Gimbrone, M. A., Jr., A. F. Brock, and A. I. Schafer. 1984. Leukotriene B, stimulates polymorphonuclear leukocyte adhesion to cultured vascular endothelial cells. J. Clin. Invest. 74:1552-1555.

2. Tonnesen, M. G., D. C. Anderson, T. A. Springer, A. Knedler, N. Avdi, and P. M. Henson. 1989. Adherence of neutrophils to cultured human microvascular endothelial cells: stimulation by chemotactic peptides and lipid mediators and dependence upon the Mac-1, LFA-1, p150,95 glycoprotein family. J. Clin. Invest. 83:637-646.

3. Charo, I. F. C. Yuen, H. D. Perez, and I. M. Goldstein. 1986. Chemotactic peptides modulate adherence of human polymorphonuclear leukocytes to monolayers of cultured endothelial cells. J. Immunol. 136:3412-3419.

4. Pohlman, T. H., K. A. Stanness, P. G. Beatty, H. D. Ochs, and J. M. Harlan 1986. An endothelial cell surface factor(s) induced in vitro by lipopolysaccharide, interleukin-1, and tumor necrosis factor increases neutrophil adherence by a CDw18 (LFA)-dependent mechanism. J. Immunol. 136:4548-4553.

5. Gamble, J. R., J. M. Harlan, S. J. Klebanoff, and M. A. Vadas. 1985. Stimulation of the adherence of neutrophils to umbilical vein endothelium by human recombinant tumor necrosis factor. Proc. Natl. Acad. Sci. USA. 82:86678674.

6. Smith, C. W., R. Rothlein, B. J. Hughes, M. M. Mariscalco, F. C. Schmalstieg, and D. C. Anderson. 1988. Recognition of an endothelial determinant for CD18-dependent human neutrophil adherence and transendothelial migration. J. Clin. Invest. 82:1746-1756.

7. Smith, C. W., S. D. Marlin, R. Rothlein, C. Toman, and D. C. Anderson. 1989. Cooperative interactions of LFA-1 and Mac-1 with intercellular adhesion molecule- 1 in facilitating adherence and transendothelial migration of human neutrophils in vitro. J. Clin. Invest. 83:2008-2017.

8. Lawrence, M. B., C. W. Smith, S. G. Eskin, and L. V. McIntire. 1990. Effect of venous shear stress on CD18-mediated neutrophil adhesion to cultured endothelium. Blood. 75:227-237.

9. Smith, C. W., S. D. Marlin, R. Rothlein, M. B. Lawrence, L. V. McIntire, and D. C. Anderson. 1989. Role of ICAM-1 in the adherence of human neutrophils to human endothelial cells in vitro. In Structure and Function of Molecules Involved in Leukocyte Adhesion. T. A. Springer, D. C. Anderson, R. Rothlein, and A. S. Rosenthal, editors. Springer-Verlag, Inc., New York. 170-189.

10. Zimmerman, G. A., and T. M. McIntyre. 1988. Neutrophil adherence to human endothelium in vitro occurs by CDw 18 (Mol, MAC-1/LFA-1/GP150,95) glycoprotein-dependent and independent mechanisms. J. Clin. Invest. 81:531537.

11. Pohlman, T. H., R. S. Munford, and J. M. Harlan. 1987. Deacylated lipopolysaccharide inhibits neutrophil adherence to endothelium induced by lipopolysaccharide in vitro. J. Exp. Med. 165:1393-1402.

12. Dunn, C. J., and W. E. Fleming. 1984. Increased adhesion of polymorphonuclear leukocytes to vascular endothelium by specific interaction of endogenous (interleukin-1) and exogenous(lipopolysaccharide) substances with endothelial cells "in vitro." Eur. J. Rheumatol. Inflammation. 7:80-86.

13. Pober, J. S., L. A. Lapierre, A. H. Stolpen, T. A. Brock, T. A. Springer, W. Fiers, M. P. Bevilacqua, D. L. Mendrick, and M. A. Gimbrone, Jr. 1987. Activation of cultured human endothelial cells by recombinant lymphotoxin: comparison with tumor necrosis factor and interleukin 1 species. J. Immunol. 138:33193324.

14. Broudy, V. C., J. M. Harlan, and J. W. Adamson. 1987. Disparate effects 
of tumor necrosis factor- $\alpha /$ cachectin and tumor necrosis factor- $\beta$ /lymphotoxin on hematopoietic growth factor production and neutrophil adhesion molecule expression by cultured human endothelial cells. J. Immunol. 138:4298-4302.

15. Lawrence, M. B., L. V. McIntire, and S. G. Eskin. 1987. Effect of flow on polymorphonuclear leukocyte/endothelial cell adhesion. Blood. 70:1284-1290.

16. Bevilacqua, M. P., J. S. Pober, D. L. Mendrick, R. S. Cotran, and M. A Gimbrone, Jr. 1987. Identification of an inducible endothelial-leukocyte adhesion molecule. Proc. Natl. Acad. Sci. USA. 84:9238-9242.

17. Bevilacqua, M. P., S. Stengelin, Jr., M. A. Gimbrone, and B. Seed. 1989. Endothelial leukocyte adhesion molecule 1: an inducible receptor for neutrophils related to complement regulatory proteins and lectins. Science (Wash. DC). 243:1160-1165.

18. Luscinskas, F. W., A. F. Brock, M. A. Arnaout, and M. A. Gimbrone, Jr. 1989. Endothelial-leukocyte adhesion molecule-1-dependent and leukocyte (CD11/CD18)-dependent mechanisms contribute to polymorphonuclear leukocyte adhesion to cytokine-activated human vascular endothelium. J. Immunol. 142:2257-2263.

19. Lasky, L. A., M. S. Singer, T. A. Yednock, D. Dowbenko, C. Fennie, H Rodriguez, T. Nguyen, S. Stachel, and S. D. Rosen. 1989. Cloning of a lymphocyte homing receptor reveals a lectin domain. Cell. 56:1045-1055.

20. Tedder, T. F., C. M. Isaacs, T. J. Ernst, G. D. Demetri, D. A. Adler, and C. M. Disteche. 1989. Isolation and chromosomal localization of cDNAs encoding a novel human lymphocyte cell surface molecule, LAM-1. Homology with the mouse lymphocyte homing receptor and other human adhesion proteins. $J$. Exp. Med. 170:123-133.

21. Kishimoto, T. K., M. A. Jutila, and E. C. Butcher. 1990. Identification of a human peripheral lymph node homing receptor: A rapidly down-regulated adhesion molecule. Proc. Natl. Acad. Sci. USA. 87:2244-2248.

22. Camerini, D., S. P. James, I. Stamenkovic, and B. Seed. 1989. Leu-8/TQ1 is the human equivalent of the Mel-14 lymph node homing receptor. Nature (Lond.). 342:78-82.

23. Tedder, T. F., A. C. Penta, H. B. Levine, and A. S. Freedman. 1990 Expression of the human leukocyte adhesion molecule, LAM-1 identity with the TQ1 and Leu-8 differentiation antigens. J. Immunol. 144:532-540.

23a.Hallmann, R., M. A. Jutila, C. W. Smith, D. C. Anderson, T. K. Kishimoto, and $\mathrm{E}$. C. Butcher. The peripheral lymph node homing receptor, LE-CAM-1, is involved in adhesion of human neutrophils to cytokine-activated endothelium in vitro. Biochem. Biophys. Res. Commun. In press.

24. Shappell, S. B., C. Toman, D. C. Anderson, A. A. Taylor, M. L. Entman, and C. W. Smith. 1990. Mac-1 (CD11b/CD18) mediates adherence-dependent hydrogen peroxide production by human and canine neutrophils. J. Immunol. 144:2702-2711.

25. Nathan, C., S. Srimal, C. Farber, E. Sanchez, L. Kabbash, A. Asch, J. Gailit, and S. D. Wright. 1989. Cytokine-induced respiratory burst of human neutrophils: dependence on extracellular matrix proteins and CD11/CD18 integrins. J. Cell Biol. 109:1341-1349.

26. Stoolman, L. M. 1989. Adhesion molecules controlling lymphocyte migration. Cell. 56:907-910.

27. Lewinsohn, D. M., R. F. Bargatze, and E. C. Butcher. 1987. Leukocyte-endothelial cell recognition: evidence of common molecular mechanism shared by neutrophils, lymphocytes, and other leukocytes. J. Immunol. 138:4313-4321.

28. Jutila, M. A., L. Rott, E. L. Berg, and E. C. Butcher. 1989. Function and regulation of the neutrophil MEL-14 antigen in vivo: comparison with LFA-1 and MAC-1. J. Immunol. 143:3318-3324.

29. Kishimoto, T. K., M. A. Jutila, E. L. Berg, and E. C. Butcher. 1989. Neutrophil Mac-1 and MEL-14 adhesion proteins inversely regulated by chemotactic factors. Science (Wash. DC). 245:1238-1241.

30. Jutila, M. A., T. K. Kishimoto, and E. C. Butcher. 1990. Regulation and lectin activity of the human neutrophil peripheral lymph node homing receptor. Blood. 75:1-5.

31. Anderson, D. C., and T. A. Springer. 1987. Leukocyte adhesion defi- ciency: an inherited defect in the Mac-1, LFA- 1 and p150,95 glycoproteins. Annu. Rev. Med. 38:175-194.

32. Entman, M. L., K. Youker, S. B. Shappell, C. Siegel, R. Rothlein, W. J. Dreyer, F. C. Schmalstieg, and C. W. Smith. 1990. Neutrophil adherence to isolated adult canine myocytes: Evidence for a CD18-dependent mechanism. $J$. Clin. Invest. 85:1497-1506.

33. Sanchez-Madrid, F., J. Nagy, E. Robbins, P. Simon, and T. A. Springer. 1983. A human leukocyte differentiation antigen family with distinct alpha subunits and a common beta subunit: the lymphocyte function associated antigen (LFA-1), the C3bi complement receptor (OKM1/Mac-1), and the p150,95 molecule. J. Exp. Med. 158:1785-1803.

34. Dana, N., B. Styrt, J. D. Griffin III, R. F. Todd, M. S. Klempner, and M. A. Arnaout. 1986. Two functional domains in the phagocyte membrane glycoproteins Mol identified with monoclonal antibodies. J. Immunol. 137:3259_ 3263.

35. Harlan, J. M., P. D. Killen, F. M. Senecal, B. R. Schwartz, E. K. Yee, R. F Taylor, P. G. Beatty, T. H. Price, and H. D. Ochs. 1985. The role of neutrophil membrane glycoprotein GP-150 in neutrophil adherence to endothelium in vitro. Blood. 66:167-178.

36. Arnaout, M. A., E. A. Wang, S. C. Clark, and C. A. Sieff. 1986. Human recombinant granulocyte-macrophage colony-stimulating factor increases cellto-cell adhesion and surface expression of adhesion-promoting surface glycoproteins on mature granulocytes. J. Clin. Invest. 78:597-601.

37. McFall, T. L., G. A. Zimmerman, N. H. Augustine, and H. R. Hill. 1987. Effect of group b streptococcal type-specific antigen on polymorphonuclear leukocyte function and polymorphonuclear leukocyte-endothelial cell interaction. Pediatr. Res. 21:517-523.

38. Breviario, F., F. Bertocchi, E. Dejana, and F. Bussolino. 1988. IL-1 induced adhesion of polymorphonuclear leukocytes to cultured human endothelial cells. J. Immunol. 141:3391-3397.

39. Schroder, J., and E. Christophers. 1989. Secretion of novel and homologous neutrophil-activating peptides by LPS-stimulated human endothelial cells. J. Immunol. 142:244-251.

40. Gimbrone, M. A., Jr., M. S. Obin, A. F. Brock, E. A. Luis, P. E. Hass, C. A Hebert, Y. K. Yip, D. W. Leung, D. G. Lowe, W. J. Kohr, et al. 1989. Endothelial interleukin-8: a novel inhibitor of leukocyte-endothelial interactions. Science (Wash. DC). 246:1601-1603.

41. Moser, R., B. Schleiffenbaum, P. Groscurth, and J. Fehr. 1989. Interleukin 1 and tumor necrosis factor stimulate human vascular endothelial cells to promote transendothelial neutrophil passage. J. Clin. Invest. 83:444-455.

42. Furie, M. B., and D. D. McHugh. 1989. Migration of neutrophils across endothelial monolayers is stimulated by treatment of the monolayers with interleukin-1 or tumor necrosis factor-a. J. Immunol. 143:3309-3317.

43. Marlin, S. D., and T. A. Springer. 1987. Purified intercellular adhesion molecule-1 (ICAM-1) is a ligand for lymphocyte function-associated antigen 1 (LFA-1). Cell. 51:813-819.

44. Kishimoto, T. K., D. C. Anderson, E. Butcher, and C. W. Smith. 1990 The human neutrophil MEL-14 antigen and the endothelial antigen ELAM-1 are involved in the same CD18-independent adhesion pathway in vitro. J. Leukocyte Biol. 48(Suppl. 1):96.

45. Imai, Y., D. D. True, M. S. Singer, and S. D. Rosen. 1990. Direct demonstration of the lectin activity of $\mathrm{gp} 90^{\mathrm{MEL}}$, a lymphocyte homing receptor. J. Cell Biol. 111:1225-1232.

46. Griffin, J. D., S. Olivier, T. J. Ernst, M. P. Belvin, H. B. Levine, Y. Karakura, and T. F. Tedder. 1990. Granulocyte-macrophage colony-stimulating factor and other cytokines regulate surface expression of the leukocyte adhesion molecule-1 on human neutrophils, monocytes, and their precursors. J. Immunol. 145:576-584.

47. Peters, W. P., A. Stuart, M. L. Affronti, C. S. Kim, R. E. Coleman. 1988. Neutrophil migration is defective during recombinant human granulocytemacrophage colony-stimulating factor infusion after autologous bone marrow transplantation in humans. Blood. 72:1310-1317. 